\title{
A Trust-Evaluation Metric for Cloud applications
}

\author{
Mohammed Alhamad, Tharam Dillon, and Elizabeth Chang
}

\begin{abstract}
Cloud services are becoming popular in terms of distributed technology because they allow cloud users to rent well-specified resources of computing, network, and storage infrastructure. Users pay for their use of services without needing to spend massive amounts for integration, maintenance, or management of the IT infrastructure. Before interaction occurs between cloud providers and users, trust in the cloud relationship is very important to minimize the security risk and malicious attacks. The notion of trust involves several dimensions. These dimensions include: the scalability, availability, security, and usability parameters of IaaS, PaaS, SaaS, and DaaS. Each of these dimensions is characterized by fuzzy aspects and linguistic terms. This paper develops a model for each of the dimensions for IaaS using fuzzy-set theory. It then uses the sugeno fuzzy-inference approach for developing an overall measure of trust value of the cloud providers. It is not easy to evaluate the cloud metrics for a general domain. So, in this paper, we will use an e-learning application as the main example when we collect the data and apply the fuzzy model to evaluate the trust for cloud computing. Test and results are presented to evaluate the effectiveness and robustness of the proposed model.
\end{abstract}

Index Terms - Trust, Cloud computing, Fuzzy inference.

\section{INTRODUCTION}

\section{A. Cloud Computing}

In the literature, various definitions of cloud computing have been proposed [1-3]. In this paper, we adopted and considered the definition provided by U.S. NIST (National Institute of Standards and Technology) that describes cloud computing as "... a model for enabling convenient, on demand network access to a share pool of configurable computing resources (e.g., networks, servers, storage, applications, and services) that can be rapidly provisioned and released with minimal management afford or service provider interaction" [1]. In other words, cloud computing is a framework by means of which virtualized infrastructure resources are delivered as a service to customers by using a public network which is the Internet [4-6]. The cloud customers can range from big organizations, small business and developers to individual users. In this paper, we will refer to such customers as 'users'. One of the advantages of having

Manuscript received July 10, 2011.

Mohammed Alhamad is with the Digital Ecosystems and Business Intelligence Institute (DEBII), Curtin University, (Mohammed.Alhamad@postgrad.curtin.edu.au).

Tharam Dillon is Distinguished Research Professor and Head of R\&D of Digital Ecosystems and Business Intelligence (DEBII), Curtin University (Tharam.Dillon@cbs.curtin.edu.au).

Elizabeth Change is Director of the Research Institute for Digital Ecosystems and Business Intelligence (DEBII), Curtin University (Elizabeth.Chang@cbs.curtin.edu.au). such a framework is that users do not need to buy costly physical infrastructure or software, but they can obtain them over a virtual environment from other users at a much lower price, thereby reducing their operational and maintenance costs. For example, Salesforce.com developed a customer relationship management solution (CRM) and delivered this as a cloud service, not as a package of software. Salesforce.com customers can use this type of service using a basic machine with an Internet browser [7]. There are four main delivery models of cloud services with such a paradigm. They are:

1. Infrastructure as a service (IaaS): In such architectures, users can use the visualization resources as a fundamental infrastructure for their applications. These resources may be a CPU, network, or storage. Cloud users can manage the resources and assign rules for end users [8].

2. Database as a service (DaaS): Such architectures allow users to rent a specific size of storage for a specific period of time. Users are not required to manage the integration or the scaling of the infrastructure. Database providers take the responsibility for integration, privacy, and security of users' data [9].

3. Platform as a service (PaaS): In these architectures, users utilize all facilities on the cloud to develop and deliver their web application and services to the end users. PaaS services may include development, integration, testing or the storage resources to complete the life cycle of services [10].

4. Software as a service (SaaS): Here, users connect with the service providers to use the application, but they do not control the infrastructure, operating system or network infrastructure $[10,11]$.

Each of these delivery models is above the required hardware and virtualization model. No matter what type of delivery model is being used, there are five essential factors or characteristics that have to be satisfied in order to achieve smooth computing in a cloud computing environment. They are:

a) On-demand self-service: This refers to the availability of the required resources (such as CPU power, network etc) as and when the user needs it. Furthermore, this should be without any human intervention [12].

b) Broad network access: As the interacting medium between the different users is the Internet, there should be a broad network access available that allows for the seamless interaction of different applications across different heterogeneous platforms [13].

c) Resource Pooling: A cloud provider should support multi-tenancy of its resources for maximising the efficiency of its infrastructure. For example, it should be able to dynamically assign the required resources to the consumer according to its demand [14]. 
d) Rapid Elasticity: It should be flexible according to the computing resources required for the customers. For example, there is no up-front commitment and the customers should be able to relinquish the resources once their work is done [15]. e) Measure of Service: There should be a framework that measures the usage of each user according to the resources that are being used by it [16].

\section{B. Trust and Fuzzy Inference System}

Trust is a very important factor in the open distributed systems. It is the main concern of all interactions between service providers and consumers in such a changing environment. The trust evaluation process is not clear and easy for diverse users because it has vague and different subjective values. This leads to the need for a clear description methodology to present the values of trust in a clear way. Fuzzy logic is an effective technique for solving this problem. The fuzzy logic approach is a better method of describing human perception. Therefore, this paper will use this approach to evaluate the trustworthiness of service providers in cloud computing.

In this paper, we will develop an approach that characterizes the key aspects of the trust relationship between cloud providers and users. Moreover, each of the trust dimensions will be represented within a fuzzy framework, and measures along each dimension will be developed. In addition, an overall figure for trust value will be developed for the cloud providers.

\section{E-learning systems}

Electronic learning is an online service that includes a wide range of e-services and applications that provide different types of online-based media to deliver fast and effective training and education. The Internet is the main tool that e-learning providers use for the delivery of complicated functions for students and school staff. E-learning systems are widely used in various sectors of teaching which include universities, companies, medical organizations, and even in training schools at a lower level. The main stockholders involved in e-learning systems are the trainers and students. Students can derive major benefits from an e-learning environment in terms of efficient media delivery time, timely feedback, communication with teachers etc. Also, students can communicate interactively with tutors and students anywhere at any time. E-learning providers can obtain more benefits by using the cloud computing infrastructure. Most of the e-learning legacy systems can be adjusted and transferred to cloud data centres to provide more scalable and available applications for students who can be anywhere in the world. In this paper, we use the e-learning system as an example of cloud applications that can be assisted and evaluated using our proposed approach which is a fuzzy logic technique. We chose an e-learning application to save the data collection time; also, there are many experts available for consultation when we define the fuzzy inputs and outputs parameters.

\section{RELATED WORK}

Different models have been proposed with fuzzy logic to provide reliable and trusted solutions for online services. For instance, Falcon et al. [17] implemented a socio-cognitive model to evaluate the trustworthiness. A Fuzzy Cognitive Map is used with different components to evaluate the impact and can be changed to suit different situations. Another model proposed by Sabater and Sierra [18] uses a fuzzy logic-based approach to analyse the relationships of the service users in electronic marketplaces. Reputation mechanisms are used in e-market systems (e.g. Amazon, E-bay) to secure the transactions of all users in a centralized architecture. Novel models of reputation and trust have been developed in e-market places to provide reliable services of security since traditional solutions to security issues do not adequately protect providers and services consumers [19]. The most important aspect of these models is the information relating to past behaviours of users. This information is used to present the reputation of those users in terms of availability, reliability, and security. As centralized architectures of online reputation models, E-bay and Amazon exemplify this approach. Their systems are implemented based on a centralized rating model so that customers and sellers can rate each other using numerical ratings or feedback comments. Users can obtain a reputation profile for a given user in order to decide whether or not to proceed with a transaction with this user. For example, E-bay uses 1, 0, -1 scales which means positive, neutral, and negative respectively. Users use these scales to rate business partners based on past behaviours. The feedback from users is stored in a central system and the reputation score is computed regularly as cumulative results of user ratings [20]. The problem with this mechanism is that users with high scores for reputation can cheat other users in the course of a few transactions even though they receive negative feedback, because these users still receive positive ratings from other customers. Also, this model cannot guarantee the consistent performance of all services from the one user. This model employs a centralized architecture; therefore, all services and reputation information have a single point of failure. Unlike the centralized architecture of service discovery, the peer-to-peer model does not use a single point to manage and store descriptions of services and reputation information. Vu et al. [8] propose peer-to-peer web service discovery that uses QoS and users' feedback to rank and select services. QoS data about services and reputation rates from consumers are stored in multi-peers in peer-to-peer systems. Monitoring agents are used to prevent cheating by users and providers. Trusted agents monitor and provide reports of services to a UDDI peer and, based on this information, services are evaluated and ranked. However, the monitoring of reports differs from peer to peer, because each peer uses different criteria to provide feedback about services. Hence, it is evident that the proposed works in trust and reputation management systems are designed mainly to enhance the security of the traditional web services. In cloud computing, the execution of services has changed to be completely independent of the consumer's infrastructure. The proposed methodology will present a novel metrics of trust for cloud computing providers. An e-learning application will be used to show the effectiveness of the proposed method when the performance evaluation is needed for cloud providers. 


\section{RESEARCH METHODOLOGY}

The research approach includes problem definition, definition of domain variables, data collection, model design, and the implementation. Figure 1 describes the research approach. The following sections describe the research steps.

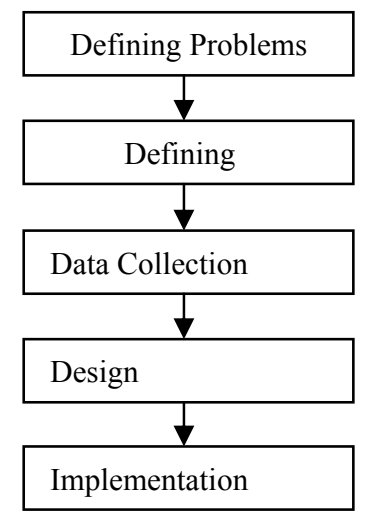

Fig. 1. The research approach.

\section{A. Problem Definition}

In the existing body of literature on cloud computing, there is no framework by which a cloud service consumer can make an intelligent trust-based decision regarding service selection from a service provider. Given the potential growth of cloud computing and the business implications, it is very important to have such architecture in place. The primary issues which are not investigated in the related literature are:

- the lack of a reliable model for trust and reputation specified for cloud architecture;

- the difficulties faced by cloud users when they want to sign online agreements with cloud providers; there is no clear and reliable method for selecting the most suitable parameters for the SLA contracts;

- the lack of a proposed model to calculate and estimate the cost for each level of the cloud architecture;

- Although trust and reputation systems have been widely proposed and implemented for various types of online services, no such models have been proposed for cloud computing; cloud users also need such systems in order to select the most trustworthy of services that are already being offered by cloud providers.

In this paper, we will focus on how to evaluate the trusted cloud providers in such a way that users of cloud can easily understand and start to build a trusting relationship with service providers.

\section{B. Defining Domain Variables}

During the process of designing the SLA model for cloud computing in our previous work [21], various parameters were investigated for cloud services as shown in Figure 2.

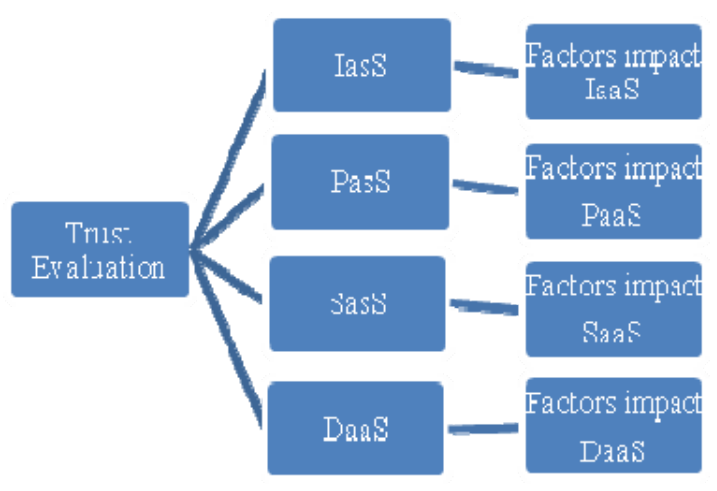

Fig. 2. Factors that impact on the different cloud services.

In this paper, we will use the main parameters which were designed for IaaS with additional parameters as the core factors that the cloud computing experts believe will impact on the trust evaluation of IaaS with an e-learning application. Table 1 lists the impact factors for trust evaluation of IaaS-based e-learning systems.

TABLE 1. FACTORS IMPACT THE TRUST VALUE OF IAAS-BASED E-LEARNING SYSTEMS

\begin{tabular}{|l|l|}
\hline Final result of trust evaluation & Factors impact trust \\
\hline \multirow{3}{*}{ Degree of trust (T) } & Scalability (Sca) \\
\cline { 2 - 2 } & Availability (Avi) \\
\cline { 2 - 2 } & Security (Sec) \\
\cline { 2 - 2 } & Usability (Usa) \\
\hline
\end{tabular}

Trust evaluation factors include scalability, availability, security, and usability. After defining the problem, this section describes the inputs and output of the proposed model. This part of the research is usually conducted after investigating the problem domain and set more relationships with the cloud computing experts. We established good relationships with a number of cloud computing experts and end users and were able to define the most important variables for our model.

\section{Data Collection}

One of the most important steps in the development of fuzzy-based control systems is the data set preparation and collection. So, the model with a fuzzy inference approach must be trained with training data that represent the greatest possibilities of application [22]. In this study, we used the data which was collected from cloud computing experts and cloud users. An online-based survey was developed in order to collect more data sets from different locations. The survey with the designed research questions was conducted to collect values for the most important variables which had already been selected to present the trust value in a cloud-based e-learning application.

\section{Design Fuzzy Model}

Fuzzy logic theory is used so as to extend the mathematics ontology in a certain method with fuzziness in order to help 
make an intelligent decision. By basing our research on the application of fuzzy logic technique to the cloud services environment, we aim to solve the problem of uncertainty in the evaluation of trust for cloud providers. The proposed fuzzy logic method in this paper uses three fuzzy sets for the input factors and five fuzzy sets for the parameters of output. The three fuzzy sets which are low (L), medium (M) and high (H) are used to characterize the fuzzy value for each input which are scalability, availability, security and usability. The fuzzy sets that represent the output parameters are: very poor $(\mathrm{VP})$, poor $(\mathrm{P})$, good $(\mathrm{G})$, very good $(\mathrm{VG})$ and excellent $(\mathrm{E})$.

The use of three fuzzy sets for four inputs as shown in Figure 3 will generate 241 fuzzy rules. This takes into consideration all possible combinations of inputs. This is a large number of rules and many of these rules are unnecessary when using the Sugeno fuzzy technique. Hence, the neural networks [23] will be used to reduce the number of fuzzy rules. So, the proposed model provides a more convenient method of evaluating the trust value of cloud providers. Table 2 shows the sample's fuzzy rules for input factors and the assigned values for output. The type of membership function for inputs and output that can be used depends on the nature of the system's attributes. In this paper, the gbell membership function is used because this is the simplest membership function that can present the input data and give a better view when we analyze the experiment results.

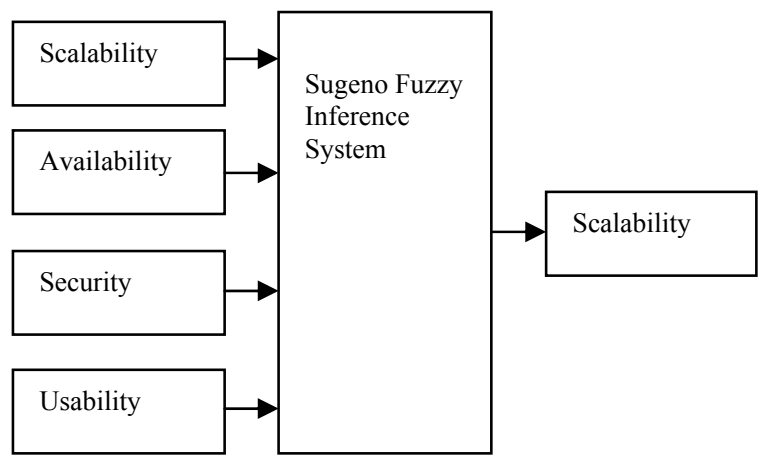

Fig. 3. Sugeno fuzzy inference model

TABLE. 2. SAMPLES OF FUZZY RULES FOR TRUST EVALUATION OF IAAS

\begin{tabular}{|c|c|c|c|c|}
\hline $\begin{array}{l}\text { IF } \\
\text { Sca }\end{array}$ & $\begin{array}{l}\text { AND } \\
\text { Avi }\end{array}$ & $\begin{array}{l}\text { AND } \\
\text { Sec }\end{array}$ & $\begin{array}{l}\text { AND } \\
\text { (Usa) }\end{array}$ & $\begin{array}{l}\text { Then } \\
\text { T }\end{array}$ \\
\hline L & L & L & L & VP \\
\hline M & L & L & M & P \\
\hline M & M & L & M & G \\
\hline L & M & M & L & P \\
\hline M & L & M & M & G \\
\hline H & L & L & H & P \\
\hline M & M & M & M & G \\
\hline H & H & L & H & G \\
\hline L & H & H & L & G \\
\hline H & L & H & H & VG \\
\hline H & M & M & H & G \\
\hline M & H & M & M & G \\
\hline H & M & H & H & VG \\
\hline
\end{tabular}

The proposed system will help cloud users to make intelligent decision in simple method. Figure 4 explain the main process for trust decision making for cloud providers.

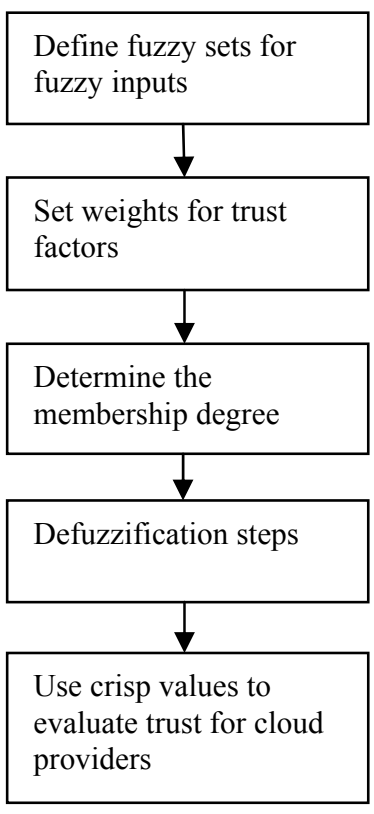

Fig. 4. Trust decision making process for cloud providers

The basic processes for the proposed model are explained as follows:

1) The first step of the proposed method is defining the fuzzy sets for all factors and fuzzy sets for the output which is the trust value for cloud providers. Then, requesters for trustworthiness about a service provider can set the weights for each factor; this step depends on the application of cloud services. If requesters do not like to provide these weights, the proposed fuzzy system will deal with all factors equally. Figure 5 describes the processes of the proposed system.

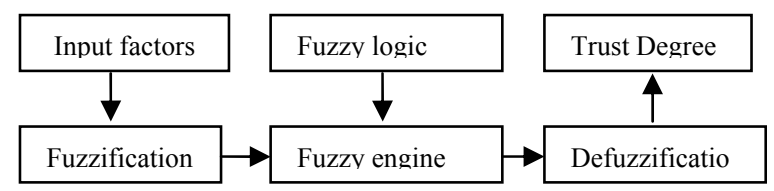

Fig..5. Fuzzy model processes

2) The second step is the fuzzification. In this part of the process, all inputs are assigned to the appropriate degree of the fuzzy sets of input. This process uses a membership function to determine the degree of input to the fuzzy set.

3) Select the fuzzy membership function. Membership function is a function that determines how each of the values in the input range belong to the input space of the membership value. The membership range is between 0 and 1. Figure 6 presents one of the membership functions of the scalability factor.

4) Design fuzzy rules. In this paper, we select the most important rules of the inference system based on the neural networks [23]. Discussions and an online survey are used to determine the means of selecting fuzzy rules that give our system more accurate and 
better performance.

5) Sugeno fuzzy inference engine. The mean technique which is used in the proposed model is the Sugeno fuzzy inference method. Sugeno's method is one of the most popular control approaches which uses the fuzzy theory. The Sugeno fuzzy inference takes the fuzzy set of inputs to produce a final output value as a crisp value..

6) Defuzzification. This type of fuzzy method uses centroid calculation in the process of defuzzification to produce a single output value.

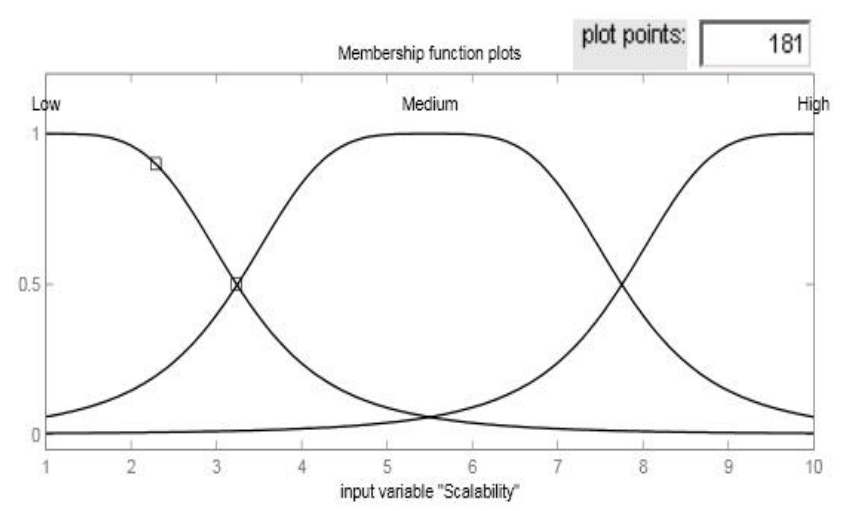

Fig. 6. Membership function of the scalability factor

\section{EXPERIMENT}

This section shows our methodology for verifying the proposed trust calculation model for cloud-based online services. In this section, the implementation of the proposed model is provided with the final results of the experiment. The fuzzy logic toolbox of Matlab is used to design and implement our model. This toolbox includes ready functions and calculation methods to implement more than one type of fuzzy inference systems such as the Mamdani and Sugeno inference system. In our model, we use the Sugeno fuzzy method with gbell membership function for inputs and output Figure 7 presents the main model for the fuzzy logic system. We used FIS editor in Matlab to develop the model. The proposed model was implemented with four input factors: scalability, availability, security, and usability. These four inputs are directed as inputs to the fuzzy inference system implemented with the Sugeno method.

Using the FIS editor, we have trained the system with 54 datasets among the 81 datasets which were collected for this experiment. Figure 8 shows the system after the training process.

In the training process, we undertake two main steps. The first step is to learn the model structure. IF-THEN rules and the knowledge from experts are used to learn the system in the first step. In the second step, we use membership functions and select the related rules to learn the parameters of inputs. Figure 9 shows the proposed system after the training process using two training steps.

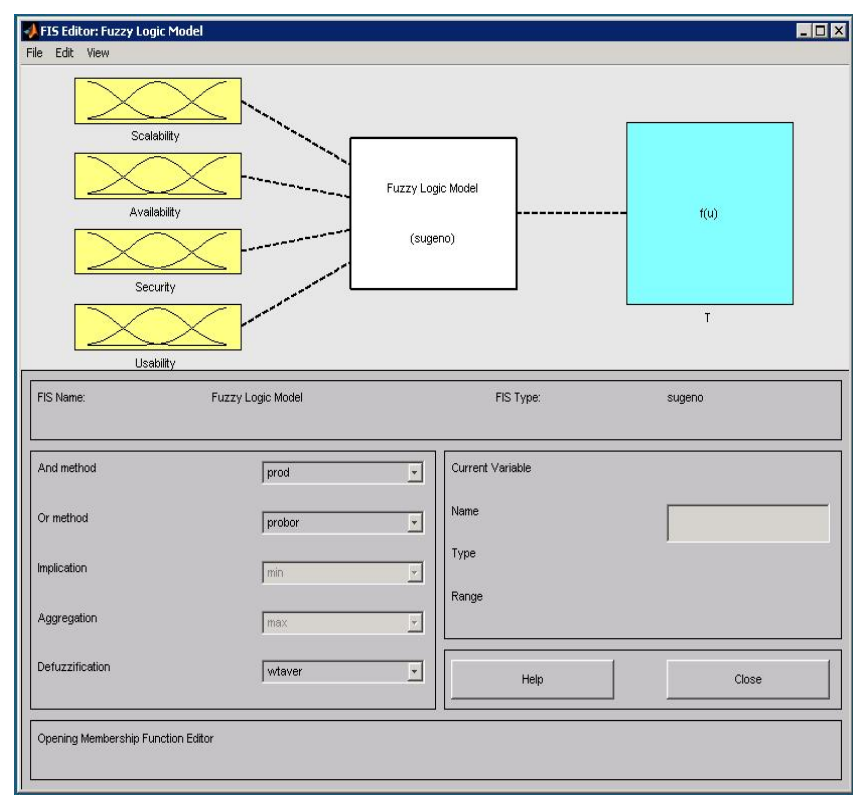

Fig. 7. FIS editor interface.
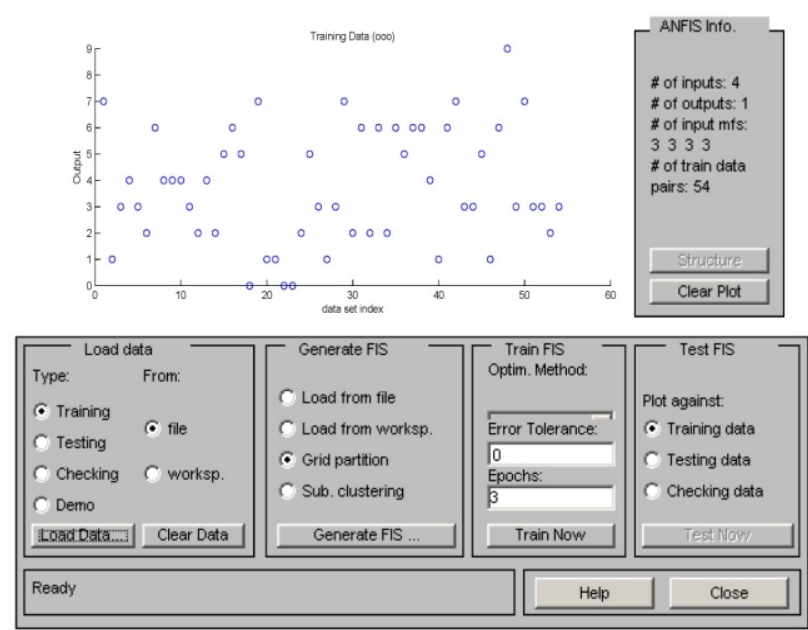

Fig. 8. Training data sets

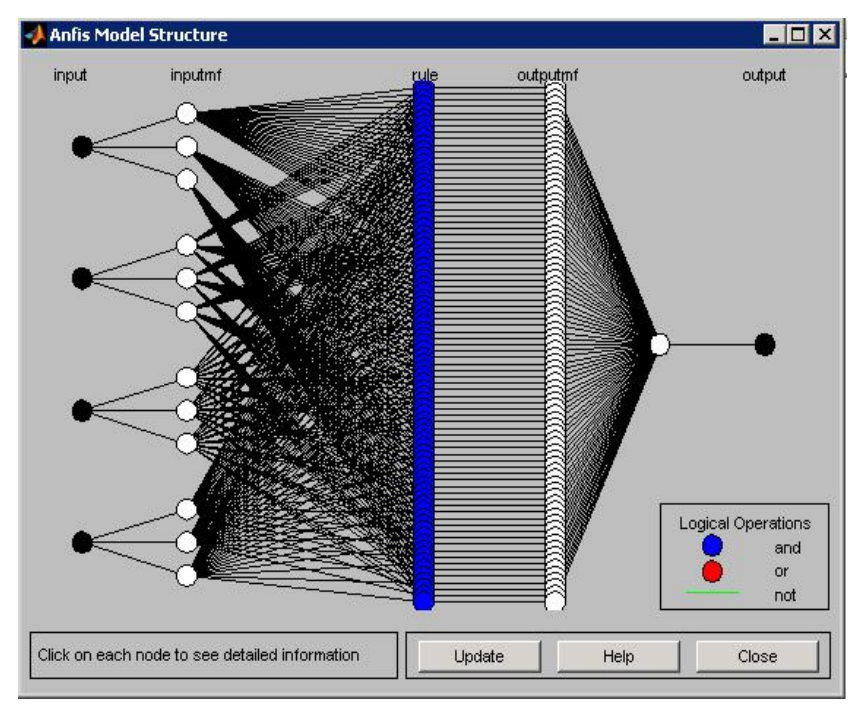

Fig. 9. Fuzzy inference system after training steps. 


\section{CONCLUSION}

In this paper, a trust evaluation scheme based on fuzzy logic system is described. The proposed scheme enables cloud users to evaluate the trustworthiness of cloud services providers when creating or shifting their distributed systems to cloud data centres. Our evaluation method uses the cloud-based e-learning system with certain factors such as security and availability as an example of a cloud-based service. We believe the proposed model provides a valid method, since the obtained results of the experiments are close to the model output using the real data sets. This fuzzy-based model can be extended for use with additional input factors. Moreover, our model can also be extended to various web-based applications such as e-commerce applications etc.

\section{REFERENCES}

[1] P. Mell and T. Grance, "Draft nist working definition of cloud computing," Referenced on June. 3rd, 2009.

[2] J. Napper and P. Bientinesi, "Can cloud computing reach the top500?," 2009, pp. 17-20.

[3] Y. Chen, et al., "What's New About Cloud Computing Security?," 2010.

[4] R. Buyya, "Market-Oriented Cloud Computing: Vision, Hype, and Reality of Delivering Computing as the 5th Utility," 2009, p. 1.

[5] A. Marinos and G. Briscoe, "Community cloud computing," CoRR, abs/0907.2485, 2009

[6] P. T. Jaeger, et al., "Cloud computing and information policy: Computing in a policy cloud?," Journal of Information Technology \& Politics, vol. 5, pp. 269-283, 2008.

[7] M. Nelson, "Building an Open Cloud," Science, vol. 324, p. 1656, 2009.
[8] D. Hilley, "Cloud Computing: A Taxonomy of Platform and Infrastructure-level Offerings," 2009.

[9] H. Cai, et al., "Customer Centric Cloud Service Model and a Case Study on Commerce as a Service."

[10] Cerbelaud, et al., "Opening the clouds: qualitative overview of the state-of-the-art open source VM-based cloud management platforms," 2009, pp. 1-8.

[11] J. Muller, et al., "Customizing Enterprise Software as a Service Applications: Back-End Extension in a Multi-tenancy Environment," 2009 , p. 66

[12] B. Sotomayor, et al., "Virtual infrastructure management in private and hybrid clouds," IEEE Internet Computing, vol. 13, pp. 14-22, 2009.

[13] D. Nurmi, et al., "The eucalyptus open-source cloud-computing system," 2009, pp. 124-131.

[14] M. Zeller, et al., "Open standards and cloud computing: Kdd-2009 panel report," 2009, pp. 11-18.

[15] T. Dillon, et al., "Cloud Computing: Issues and Challenges," 2010, pp. 27-33.

[16] J. Nunamaker Jr, et al., "Systems development in information systems research," Journal of Management Information Systems, pp. 89-106, 1990.

[17] R. Falcone, et al., "A fuzzy approach to a belief-based trust computation," Trust, reputation, and security: theories and practice, pp. $55-60,2003$

[18] J. Sabater and C. Sierra, "Reputation and social network analysis in multi-agent systems," 2002, pp. 475-482.

[19] T. Grandison and M. Sloman, "A survey of trust in internet applications," IEEE Communications Surveys and Tutorials, vol. 3, pp. 2-16, 2000.

[20] P. Resnick and R. Zeckhauser, "Trust among strangers in Internet transactions: Empirical analysis of eBay's reputation system," Advances in Applied Microeconomics: A Research Annual, vol. 11, pp. 127-157, 2002.

[21] A. Mohammed, et al., "Response Time for Cloud Computing Providers," presented at the IIWAS, Paris.

[22] C. Yin, et al., "Methods to improve prediction performance of ANN models," Simulation Modelling Practice and Theory, vol. 11, pp. 211-222, 2003.

[23] S. Sestito and T. Dillon, "Automated knowledge acquisition," 1994. 